\title{
Corner transfer matrices in statistical mechanics
}

\author{
R. J. Baxter \\ Centre for Mathematics and its Applications, The Australian National University, \\ Canberra, ACT 0200, Australia \\ E-mail: none
}

November 7th, 2006

\begin{abstract}
Corner transfer matrices are a useful tool in the statistical mechanics of simple two-dimensinal models. They can be very effective way of obtaining series expansions of unsolved models, and of calculating the order parameters of solved ones. Here we review these features and discuss the reason why the method fails to give the order parameter of the chiral Potts model.
\end{abstract}

PACS numbers 64.60.Cn, 05.50.+q

Mathematics Subject Classification: 82B20, 82B23

\section{Introduction}

In an early work of the author, [1] he evaluated numerically a sequence of approximations for the free energy (or entropy) of the monomer-dimer system on the square lattice, using a variational approximation for the eigenvalues and eigenvectors of the row-to-row transfer matrix. His interest was to see if the model exhibited a phase transition as the density of dimers increased from zero to the close packed value of one-half. No such transition was observed: the model is critical only at close packing, where it can be solved exactly[20]. 
This was reflected in the poorer convergence of the approximations as close packing is approached.

The result may have been negative, but the work did lead naturally to the development of the useful concept of "corner transfer matrices".

These matrices build up the lattice by rotations about the centre. For an infinite lattice they are of infinite dimension, but for the zero-field Ising model, one can write them in terms of the spinor operators (or Clifford algebra) introduced by Kaufman[21] and diagonalize them exactly. 2, 25] The results were surprisingly simple, and provided a reasonably direct way of obtaining the spontaneous magnetization (the order parameter) of the Ising model, compared with the previous verifications of the Onsager-Kaufman result [23] by Yang, 26] Montroll, Potts and Ward[22] and others.

It was also realised that the corner transfer matrices satisfy certain equations. Again, to give exact results the matrices and equations must be infinite-dimensional, but a finite truncation corresponds to a variational approximation of the type used for the monomer-dimer system. Such truncations can give useful approximations to the exact results, even for models which have not been solved exactly. For instance, Baxter and Enting[12] were able to obtain 24 terms in the low-temperature series expansion of the Ising model in a field, using only 15 by 15 matrices. This was a great improvement on the 12-term series expansions previously obtained. 24]

Here we present these equations for a quite general "edge-interaction" model, where spins live on sites of the square lattice and the interactions are between adjacent spins. We discuss the simplifications that arise for a "solvable" model, i.e. one whose Boltzmann weights satisfy the star-triangle relation. We emphasize the importance of the "rapidity-difference" property and indicate how it ensures that the corner transfer matrices commute and have a very simple eigenvalue spectrum. This makes it easy to obtain the order parameters of such models.

Finally we discuss the chiral Potts model, and indicate how the lack of a rapidity difference property for that model prevents its solution by the same corner transfer matrix techniques.

For definiteness, here we consider only $Z_{N}$-invariant models (such as the Ising, self-dual Potts and chiral Potts models), where each spin can take $N$ values and the Boltzmann weights depend only on the difference (modulo $N$ ) of the spin values. The extension to more general edge-interaction models (perhaps including site weight functions as in the solvable Kashiwara-Miwa model[19] - [15]) is straightforward. 


\section{Square lattice edge-interaction models}

In Figure 1 we have drawn the square lattice $\mathcal{L}$ diagonally, denoting sites by circles and edges by solid lines. On each site $i$ there is a "spin" $\sigma_{i}$, taking some discrete set of $N$ values, say $0,1, \ldots, N-1$. Spins on adjacent sites $i$ and $j$, with $j$ above and to the right of $i$, interact with Boltzmann weight function $W\left(\sigma_{i}-\sigma_{j}\right)$, as indicated. Similarly, spins on sites $k$ and $l$, with $l$ above and to the left of $k$, interact with weight function $\bar{W}\left(\sigma_{k}-\sigma_{l}\right)$. The partition function is

$$
Z=\sum_{\sigma} \prod_{i, j} W\left(\sigma_{i}-\sigma_{j}\right) \prod_{k, l} \bar{W}\left(\sigma_{k}-\sigma_{l}\right),
$$

the sum being over all values of all the spins on the lattice, the first product over all SW - NE edges, the second over all SE - NW edges.

If the lattice has $L$ sites, we expect the limit

$$
\kappa=\lim _{L \rightarrow \infty} Z^{1 / L}
$$

to exist, and to be independent of the manner in which $\mathcal{L}$ becomes large, so long as it becomes infinite in all directions.

In Figure 1 we have singled out a central site with spin $a$. The average of any function $f(a)$ is defined as

$$
\langle f(a)\rangle=Z^{-1} \sum_{\sigma} f(a) \prod_{i, j} W\left(\sigma_{i}-\sigma_{j}\right) \prod_{k, l} \bar{W}\left(\sigma_{k}-\sigma_{l}\right) .
$$

We also expect any such average to tend to a limit, provided we impose suitable conditions on the values of the boundary spins, and take a limit where $a$ becomes infinitely far from any boundary.

\subsection{Corner transfer matrices}

Here we define the corner transfer matrices (and the associated row and column matrices) for a general anisotropic edge-interaction model.

We have again drawn the diagonal square lattice in Fig. 2 and have singled out a central edge linking two sites with spins $a$ and $b$. We have then used dotted lines to divide the lattice into nine parts: the central edge, four "corners" labelled respectively $A_{1}^{a}, B_{2}, A_{3}^{b}, B_{4}$, and four columns or rows $F_{1}^{a}, G_{2}^{b}, F_{3}^{b}, G_{4}^{a}$. 


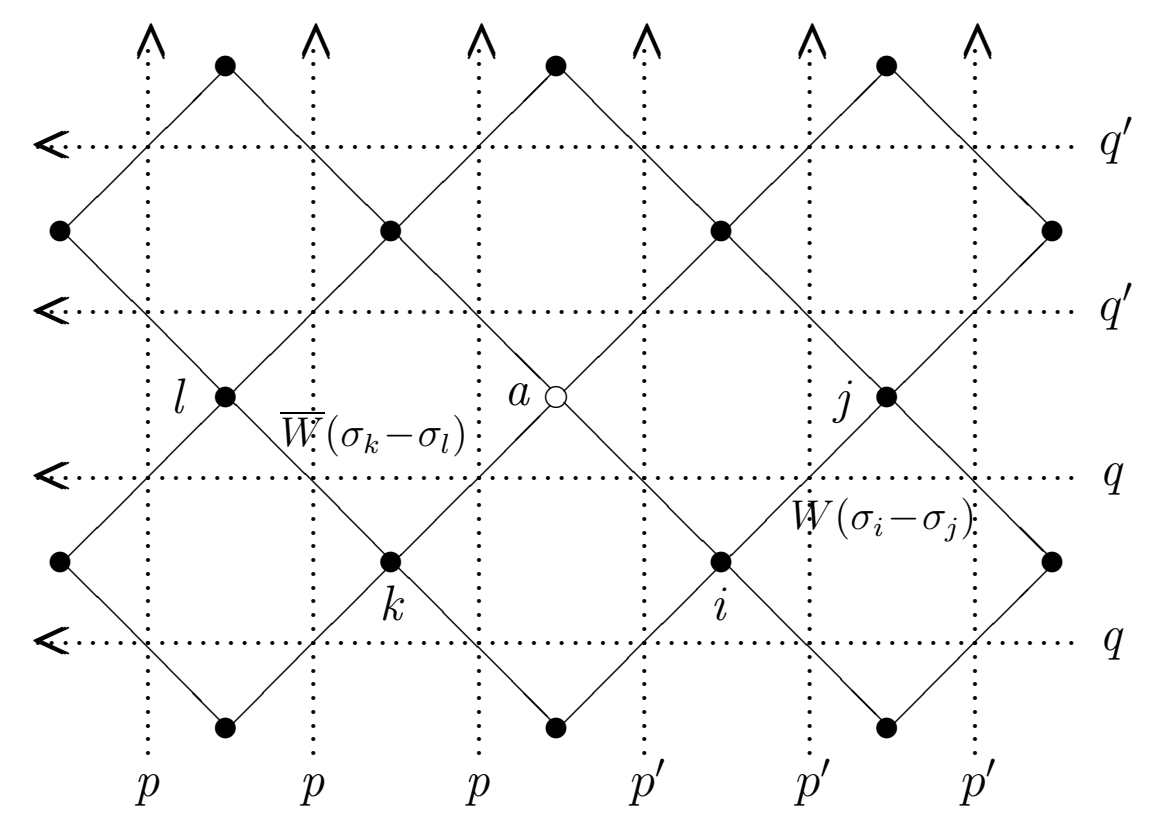

Figure 1: The square lattice (solid lines, drawn diagonally), showing a central spin $a$ and the (dotted) rapidity lines.

Take all the spins on the outermost boundary of the lattice to have some fixed value 1 say zero. Let $\lambda$ be the set of spins on the dotted line to the left of the spin $a$ and $\mu$ the set of spins on the dotted line below $a$. Consider all the edges of the lattice below $\lambda$ and to the left of $\mu$, i.e. all the edges in the lower-left corner of the lattice. Take the product over all such edges of the appropriate weight functions $W$ or $\bar{W}$, and sum over all the spins internal to that corner. The resulting partition function of the corner will be a function of $a, \lambda$ and $\mu$. Write it as $\left(A_{1}^{a}\right)_{\lambda, \mu}$.

Similarly, let $\left(F_{1}^{a}\right)_{\mu, \nu}$ be the product of the weight functions of the edges between $\mu$ and $\nu$. Let $\left(B_{2}\right)_{\nu, \tau}$ be the partition function of the lower-right corner of the lattice, i.e. the portion to the right of $\mu$ and below $\tau$, and so on. Then the partition function of the complete lattice is $Z=Z_{W}$, where

$$
Z_{W}=\sum W(a-b)\left(A_{1}^{a}\right)_{\lambda, \mu}\left(F_{1}^{a}\right)_{\mu, \nu}\left(B_{2}\right)_{\nu, \tau} \ldots\left(G_{4}^{a}\right)_{\rho, \lambda},
$$

\footnotetext{
${ }^{1}$ For an ordered system, the boundary spins should be set to values consistent with the ground state of the particular phase under consideration.
} 


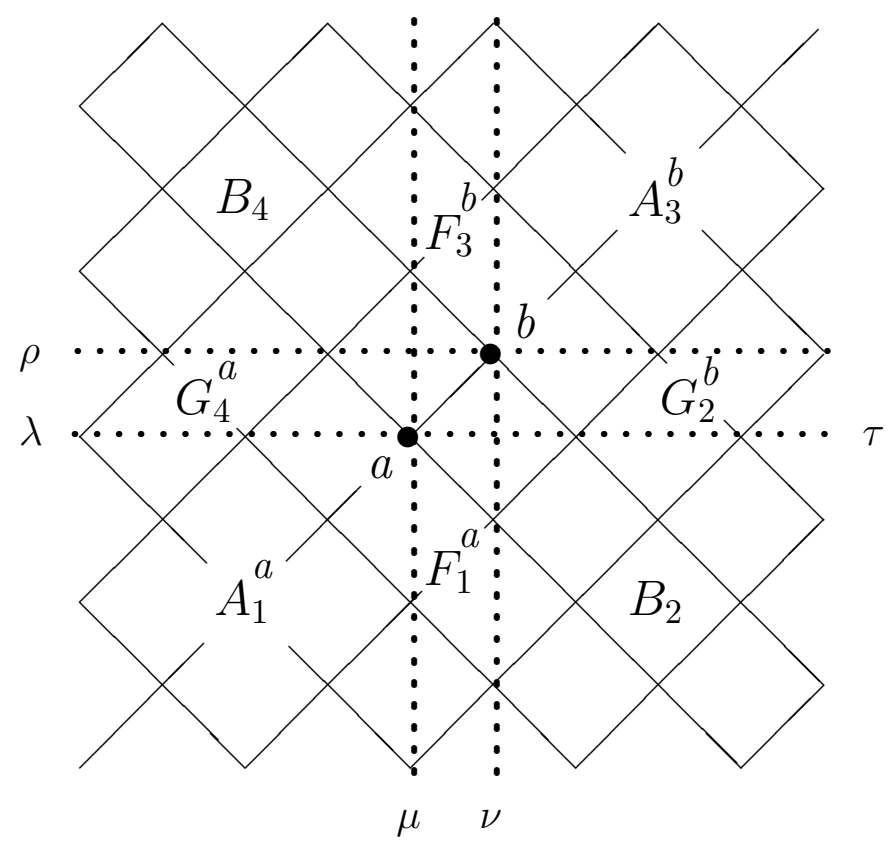

Figure 2: The parts of the lattice corresponding to the various matrices $A, B, F, G$.

the sum being over all values of $a, b$ and all the spins on the dotted lines of Fig. 2.

Plainly we can regard $\left(A_{1}^{a}\right)_{\lambda, \mu}$ as the element $(\lambda, \mu)$ of a matrix $A_{1}^{a},\left(F_{1}^{a}\right)_{\mu, \nu}$ as the element $(\mu, \nu)$ of a matrix $F_{1}^{a}$, etc. Then (2.4) simplifies to

$$
Z_{W}=\sum_{a, b} W(a-b) \text { Trace } A_{1}^{a} F_{1}^{a} B_{2} G_{2}^{b} A_{3}^{b} F_{3}^{b} B_{4} G_{4}^{a},
$$

the sum now being only over the spins $a, b$.

There are other ways of building up $Z$ using the matrices $A_{i}, B_{i}, F_{i}, G_{i}$ as building blocks, most of them being simpler than (2.5). They are

$$
\begin{gathered}
Z_{\bar{W}}=\sum_{a, b} \bar{W}(a-b) \operatorname{Trace} B_{1} G_{1}^{a} A_{2}^{a} F_{2}^{a} B_{3} G_{3}^{b} A_{4}^{b} F_{4}^{b}, \\
Z_{A}=\sum_{a} \operatorname{Trace} A_{1}^{a} A_{2}^{a} A_{3}^{a} A_{4}^{a}, Z_{B}=\operatorname{Trace} B_{1} B_{2} B_{3} B_{4},
\end{gathered}
$$




$$
Z_{i}=\sum_{a} \operatorname{Trace} A_{i}^{a} F_{i}^{a} B_{i+1} B_{i+2} G_{i+2}^{a} A_{i+3}^{a}
$$

where $i=1, \ldots, 4$ and the suffixes containing $i$ are to be interpreted modulo 4. We have indicated how $Z_{A}$ and $Z_{2}$ are constructed in Fig. 3 ,

Note that $F_{i}^{a}$ always follows $A_{i}^{a}$ and precedes $B_{i+1}$, while $G_{i}^{a}$ follows $B_{i}$ and precedes $A_{i+1}^{a}$.
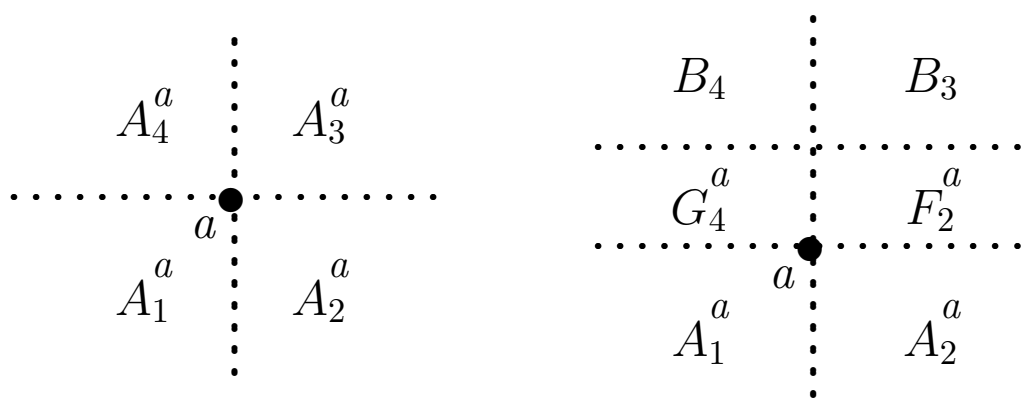

Figure 3: The partition functions $Z_{A}, Z_{2}$. We have omitted the edges of $\mathcal{L}$, but their positions can be deduced from the full circle denoting the lattice spin $a$.

If we keep the sizes of the blocks corresponding to the $A_{i}, B_{i}, F_{i}, G_{i}$ matrices fixed (and mutually consistent), then $Z_{A}, Z_{B}$ correspond to the smallest lattices, $Z_{W}, Z_{\bar{W}}$ to the largest, and $Z_{1}, \ldots Z_{4}$ to intermediate ones. Using (2.2), we find that in the limit of the lattices becoming large, the partition function per site is

$$
\kappa=\frac{Z_{A} Z_{B} Z_{W} Z_{\bar{W}}}{Z_{1} Z_{2} Z_{3} Z_{4}}
$$

We shall find it convenient to define four Boltzmann weight functions 
$U_{1}, \ldots, U_{4}$ by

$$
\begin{aligned}
& U_{1}(n)=W(n) \quad, \quad U_{2}(n)=\bar{W}(n), \\
& U_{3}(n)=W(-n) \quad, \quad U_{4}(n)=\bar{W}(-n)
\end{aligned}
$$

and to set

$$
\tilde{Z}_{1}=\tilde{Z}_{3}=Z_{W} \quad, \quad \tilde{Z}_{2}=\tilde{Z}_{4}=Z_{\bar{W}} .
$$

The equations [3, 4] for the corner transfer matrices can be obtained by requiring that the expression (2.6) be stationary with repect to variations in the matrices $A_{i}, B_{i}, F_{i}, G_{i}$. This variational principle leads to the four equations

$$
\begin{gathered}
\sum_{b} U_{i}(a-b) G_{i+1}^{b} A_{i+2}^{b} F_{i+2}^{b} B_{i+3} G_{i+3}^{a}=\eta_{i} B_{i+2} G_{i+2}^{a} A_{i+3}^{a}, \\
\sum_{b} U_{i-1}(a-b) F_{i-1}^{a} B_{i} G_{i}^{b} A_{i+1}^{b} F_{i+1}^{b}=\eta_{i}^{\prime} A_{i}^{a} F_{i}^{a} B_{i+1}, \\
F_{i}^{a} B_{i+1} B_{i+2} G_{i+2}^{a}=\xi_{i} A_{i+1}^{a} A_{i+2}^{a}, \\
\sum_{b} G_{i-2}^{b} A_{i-1}^{b} A_{i}^{b} F_{i}^{b}=\xi_{i}^{\prime} B_{i-1} B_{i} .
\end{gathered}
$$

Our notation is now such that rotating the lattice through $90^{\circ}$ is equivalent to incrementing $i$ by 1 in all suffixes.

Pre-multiplying both sides of (2.9a) by $A_{i}^{a} F_{i}^{a} B_{i+1}$ and summing over $a$, we find that

$$
\eta_{i}=\tilde{Z}_{i} / Z_{i}
$$

Similarly,

$$
\eta_{i}^{\prime}=\tilde{Z}_{i+1} / Z_{i}, \quad \xi_{i}=Z_{i} / Z_{A}, \quad \xi_{i}^{\prime}=Z_{i} / Z_{B}
$$

and, for instance,

$$
\kappa=\eta_{i} \eta_{i+2}^{\prime} /\left(\xi_{i+1} \xi_{i+3}^{\prime}\right) .
$$

The equations (2.9a) - (2.9d) can be represented graphically, each side being a semi-lattice, e.g. for $i=3$ the equation (2.9a) can be represented as in Fig. 4 .

We can only expect these equations to be exact (for an infinite system) when the matrices are infinitely dimensional. However, if we take them to finite-dimensional, then the fact that (2.9a) - (2.9d) are derived from the 


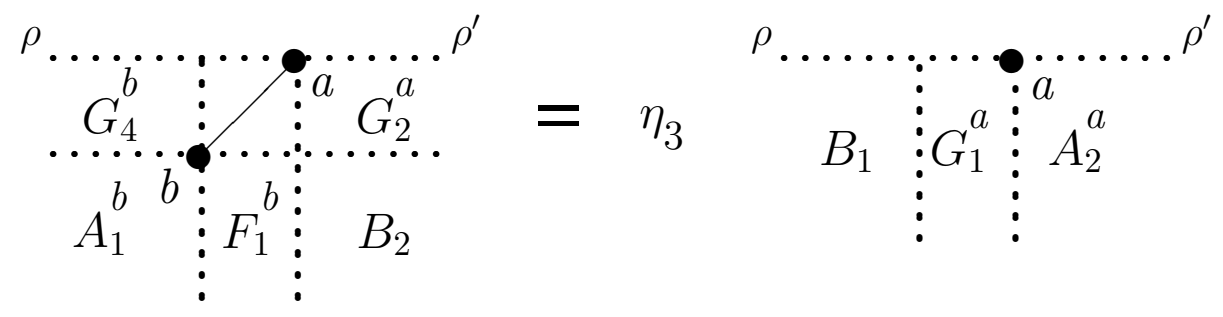

Figure 4: Graphical representation of the elements $\left(\rho, \rho^{\prime}\right)$ of the two sides of equation (2.9a) for $i=3$.

variational principle for $\kappa$ ensures that these equations are mutually consistent and that they will define the $A_{i}, B_{i}, F_{i}, G_{i}$ (to within irrelevant similarity transformations). There will be many solutions - a test of the utility of any particular solution is that it should reproduce as many of the largest eigenvalues of of $A_{1}^{a} A_{2}^{a} A_{3}^{a} A_{4}^{a}$ and $B_{1} B_{2} B_{3} B_{4}$ as possible for that truncation.

Such truncations can provide a powerful tool for performing numerical or series expansion calculations on otherwise unsolved models. [9] Indeed, it was such a calculation that led to the exact solution of the hard hexagon model.[14, [5]

\section{Solvable models}

We now focus our attention on models that are known to be solvable and satisfy the star-triangle relation. Their corner transfer matrix have special simplifying properties. These properties are true only in the infinitedimensional limit, but for any given truncation they will be approximately true, in particular they will hold to appropriate orders in a low-temperature series expansion. 
In general, if a model satisfies the star-triangle relation, then its weights $W(n), \bar{W}(n)$ depend on two variables $p$ and $q$, called "rapidities". The variable $p$ is assocaited with the vertical direction of the lattice $\mathcal{L}, q$ with the horizontal direction. We display this dependence by writing the weights as $W_{p q}(n), \bar{W}_{p q}(n)$. The star-triangle relation relates three sets of Boltzmann weights, with rapidities $(p, q),(p, r),(q, r)$. It is

$$
\begin{aligned}
\sum_{d} \bar{W}_{q r}(b-d) W_{p r} & (a-d) \bar{W}_{p q}(d-c) \\
& =R_{p q r} W_{p q}(a-b) \bar{W}(b-c) W_{q r}(a-c),
\end{aligned}
$$

for all $p, q, r$ and values of the three external spins $a, b, c$. The sum is over all values of the internal spin $d$.

A second relation must also hold, where all the spin-difference arguments in (3.13) are negated, so that $b-d$ becomes $d-b, a-b$ becomes $b-a$, etc. Provided we impose cyclic boundary conditions, (3.13) ensures that the rowto-row transfer matrices of two models, one with rapidities $(p, q)$, the other with $(p, r)$, commute. 4] Thus it is natural to allow $q, r$ to vary from row to row of the lattice $\mathcal{L}$, and in Fig. 1 we have used this freedom to associate two rapidities $q, q^{\prime}$ with the horizontal rows of the lattice. Moreover, this is true even if the rapidities on the dotted vertical lines of Fig. 1 are different, e.g. if the lines to the left of $a$ have rapidity $p$, while those to the right have rapidity $p^{\prime}$,as shown.

More generally, we can allow a different rapidities $p$ for each of the dotted vertical lines in Fig. 11, and a different rapidity $q$ for each horizontal line. The Boltzmann weight of any edge of $\mathcal{L}$ is $W_{p q}(n)$ or $\bar{W}_{p q}(n)$, where the $p$ and $q$ are the rapidities of the dotted lines intersecting that edge.

It follows that the eigenvectors of these transfer matrices are independent of horizontal rapidities such as the $q, q^{\prime}$ shown in Fig. 1. The correlations of spins lying within a single row of $\mathcal{L}$ will therefore also be independent of $q, q^{\prime}$.

But (to within an overall irrelevant normalization factor ) the expressions represented by Fig. 4 are precisely such correlations, involving only the spin sets $\rho, \rho^{\prime}$ and the spin $a$ on the upper edge of each side. This implies that

$$
\left(B_{1} G_{1}^{a} A_{2}^{a}\right)_{\rho, \rho^{\prime}}=\text { independent of } q, q^{\prime} .
$$

There is a problem with this argument: we are using fixed-spin boundary conditions, not cyclic ones. However, provided the conditions are commensurate with the ground state of the system, we expect (3.14) to be true in 
the limit when the lattice is large and only a finite number of the spins in $\rho, \rho^{\prime}$ differ from the ground-state values. This is how we construct the infinite-dimensional matrices, so we believe (3.14) to be true in this limit.

For $i$ odd, it follows that the matrices

$$
A_{i}^{a} A_{i+1}^{a}, B_{i} B_{i+1}, A_{i}^{a} F_{i}^{a} B_{i+1}, B_{i} G_{i}^{a} A_{i+1}^{a}
$$

are independent of horizontal rapidities such as $q, q^{\prime}$. Similarly,for $i$ even, they are independent of vertical rapidities such as $p, p^{\prime}$.

Each matrix will depend only on the rapidities $p, q$ of that particular, corner, row or column. For instance $B_{1}$ will depend only on the rapidities of the lower-left corner. For each matrix we take all the $p$ 's to be that same,and all the $q$ 's, and write the matrix as a function of $p, q$, e.g.

$$
B_{1}=B_{1}(p, q) \text {. }
$$

For $i$ odd, each of the expressions (3.15) is a product of matrices corresponding to blocks of $\mathcal{L}$ that occupy the same rows of $\mathcal{L}$. The horizontal rapidity $q$ must therefore be the same for each matrix within the expression, but the vertical $p$ rapidities may be different. Thus for instance the above argument gives

$$
B_{1}(p, q) B_{2}\left(p^{\prime}, q\right)=\text { independent of } q
$$

for all $p, p^{\prime}, q$. Fixing $p^{\prime}$ and assuming the $B_{i}$ matrices to be invertible, it follows that there exist invertible matrices $R(p), S(q)$ such that

$$
B_{1}(p, q)=R(p) S(q) .
$$

This is a factorization property. All the corner matrices $A_{i}^{a}, B_{i}$ similarly factor and we find that

$$
\begin{aligned}
A_{i}^{a}(p, q) & =\left[X_{i-1}^{a}\left(\tilde{p}_{i}\right)\right]^{-1} \mathcal{A}_{i}^{a} X_{i}^{a}\left(\tilde{q}_{i}\right), \\
B_{i}(p, q) & =\left[Y_{i-1}\left(\tilde{p}_{i}\right)\right]^{-1} \mathcal{B}_{i} Y_{i}\left(\tilde{q}_{i}\right), \\
F_{i}^{a}(p, q) & =\left[X_{i}^{a}\left(\tilde{q}_{i}\right)\right]^{-1} \mathcal{F}_{i}^{a}\left(\tilde{p}_{i}\right) Y_{i}\left(\tilde{q}_{i}\right), \\
G_{i}^{a}(p, q) & =\left[Y_{i}\left(\tilde{q}_{i}\right)\right]^{-1} \mathcal{G}_{i}^{a}\left(\tilde{p}_{i}\right) X_{i}^{a}\left(\tilde{q}_{i}\right),
\end{aligned}
$$

where $\tilde{p}_{i}=p, \tilde{q}_{i}=q$ if $i$ is odd; and $\tilde{p}_{i+1}=\tilde{q}_{i}, \tilde{q}_{i+1}=\tilde{p}_{i}$ for all $i$. Note that the matrices $\mathcal{A}_{i}^{a}, \mathcal{B}_{i}$ are independent of $p$ and $q$, while $\mathcal{F}_{i}^{a}, \mathcal{G}_{i}^{a}$ depend only on the single rapidity $\tilde{p}_{i}$. 
The choice of the $X_{i}, Y_{i}$ is not unique: each can be pre-multiplied by different constant invertible matrices $C_{i}, D_{i}$ and the $\mathcal{A}_{i}, \mathcal{B}_{i}, \mathcal{F}_{i}, \mathcal{G}_{i}$ then adjusted accordingly so as to ensure that (3.16) remains satisfied. However, this does not change the eigenvalues of

$$
\mathcal{M}^{a}=\mathcal{A}_{1}^{a} \mathcal{A}_{2}^{a} \mathcal{A}_{3}^{a} \mathcal{A}_{4}^{a}, \mathcal{N}=\mathcal{A}_{1} \mathcal{A}_{2} \mathcal{A}_{3} \mathcal{A}_{4}
$$

These matrices define $Z_{A}$ and $Z_{B}$ and play an imortant role in the calculations: for instance, the order parameters are

$$
\left\langle\omega^{j a}\right\rangle=\sum_{a} \omega^{j a} \text { Trace } \mathcal{M}^{a} / \sum_{a} \text { Trace } \mathcal{M}^{a}
$$

where $\omega=\mathrm{e}^{21 \pi / N}$ and $j=1, \ldots, N-1$.

Substituting these forms into the equations (2.9a) - (2.9d), all the $X_{i}, Y_{i}$ matrices cancel out, so the effect is to replace the matrices $A_{i}, B_{i}, F_{i}, G_{i}$ by $\mathcal{A}_{i}, \mathcal{B}_{i}, \mathcal{F}_{i}, \mathcal{G}_{i}$, where $\mathcal{A}_{i}, \mathcal{B}_{i}$ are rapidity-independent and $\mathcal{F}_{i}, \mathcal{G}_{i}$ depend only on the single rapidity $\tilde{p}_{i}$.

\subsection{Properties and symmetries}

There are various properties and symmetries that relate the corner transfer matrices. For the Ising, self-dual Potts and chiral Potts models[13] it is true that

$$
W_{p p}(n)=1, \bar{W}_{p p}(n)=\delta_{n, 0}
$$

and there exist rotation and reflection transformations $p \rightarrow R p, p \rightarrow S p$, such that

$$
\begin{aligned}
& \bar{W}_{p q}(n)=W_{q, R p}(n), W_{p q}(n)=\bar{W}_{q, R p}(-n), \\
& W_{p q}(n)=W_{S q, S p}(n), \bar{W}_{p q}(n)=\bar{W}_{S q, S p}(-n) .
\end{aligned}
$$

If we write $U_{i}(n)$ more explicitly as $U_{i}(p, q \mid n)$, it follows that $U_{i}(q, R p \mid n)=$ $U_{i+1}(p, q \mid n)$ and

$$
\begin{gathered}
A_{i}^{a}(p, p)=B_{i}(p, p)=1 \text { for } i=1 \text { and } 3 \\
A_{i}^{a}(q, R p)=A_{i+1}^{a}(p, q), \ldots, G_{i}^{a}(q, R p)=G_{i+1}^{a}(p, q),
\end{gathered}
$$

and, for instance,

$$
B_{i}(S q, S p)=B_{6-i}(p, q)^{T}
$$


Replacing $p, q$ by $q, R p$ is simply equivalent to rotating the lattice through $90^{\circ}$, i.e. incrementing the suffixes $i$ by one.

These equations impose many restrictions of the matrices $X_{i}, Y_{i}, \mathcal{A}_{i}, \mathcal{B}_{i}$, $\mathcal{F}_{i}, \mathcal{G}_{i}$. We shall not explore all of them here, but note that they do imply that there exists a matrix function $X^{a}(p)$ such that we can choose

$$
\begin{gathered}
X_{1}^{a}(p)=X_{4}^{a}(p)=X^{a}(p), X_{2}^{a}(p)=X_{3}^{a}(p)=\left(\mathcal{A}_{2}^{a}\right)^{-1} X^{a}(p), \\
\mathcal{A}_{1}^{a}=\mathcal{A}_{3}^{a}=\mathbf{1} \\
A_{1}^{a}(p, q)=\left[X^{a}(p)\right]^{-1} X^{a}(q), A_{2}^{a}(p, q)=\left[X^{a}(q)\right]^{-1} X^{a}(R p), \\
A_{3}^{a}(p, q)=\left[X^{a}(R p)\right]^{-1} X^{a}(R q), A_{4}^{a}(p, q)=\left[X^{a}(R q)\right]^{-1} X^{a}\left(R^{2} p\right),
\end{gathered}
$$

where

$$
X^{a}\left(R^{2} p\right)=\mathcal{M}^{a} X^{a}(p)
$$

Corresponding equations hold for the matrices $Y_{i}, \mathcal{B}_{i}, B_{i}, \mathcal{N}_{i}$, with no superfixes $a$.

\section{$4 \quad$ Ising model}

We have seen how the star-triangle relation implies that row-to-row transfer matrices commute and corner transfer matrices factor. To proceed further we need an important property, namely that for many models we can choose the rapidity variables $p, q$ so that

$$
W_{p q}(n), \bar{W}_{p q}(n)=\text { functions only of } q-p \text { and } n \text {. }
$$

We call this the rapidity difference property. It is intimately connected with the fact that for such models the Boltzmann weights live on algebraic curves of genus 0 or 1 , so can be naturally be parametrized in terms of Jacobi elliptic functions. One had become so used to this property that it was taken for granted. It was the discovery of the chiral Potts model[13], which does not have this property, that made its importance obvious.

The Ising model is a two-state model (i.e. $N=2$ ) of the type discussed above. Taking $W_{p q}(0)=\bar{W}_{p q}(0)=1$, its other Boltzmann weights are [8]

$$
W_{p q}(1)=\mathrm{e}^{-2 J}=k^{\prime} \operatorname{scd}(K-u),
$$




$$
\bar{W}_{p q}(1)=\mathrm{e}^{-2 \bar{J}}=k^{\prime} \operatorname{scd}(u),
$$

where

$$
u=u_{q}-u_{p},
$$

and the function $\operatorname{scd}(\mathrm{u})$ is defined by

$$
\operatorname{scd}(u)=\operatorname{sn}(u / 2) /[\operatorname{cn}(u / 2) \operatorname{dn}(u / 2)],
$$

sn $u, \operatorname{cn} u, \operatorname{dn} u$ being the Jacobi ellptic functions of argument $u$ and modulus $k$.

Then, setting $k^{\prime}=\left(1-k^{2}\right)^{1 / 2}$, we have

$$
\sinh 2 J=\frac{\operatorname{sn} u}{\operatorname{cn} u}, \sinh 2 \bar{J}=\frac{\operatorname{cn} u}{k^{\prime} \operatorname{sn} u}
$$

and $\sinh 2 J \sinh 2 \bar{J}=1 / k^{\prime}$. The conjugate modulus $k^{\prime}$ is small at low temperatures, increasing to one at criticality. Within this range the model has ferromagnetic order. The Boltzmann weights satisfy (3.19) if we define

$$
u_{R p}=u_{p}+K,
$$

they are real and positive provided $u=u_{q}-u_{p}$ is real and

$$
0<u_{q}-u_{p}<K
$$

$K, K^{\prime}$ being the usual complete elliptic integrals. Note that this restriction remains satisfied if we replace $p, q$ by $R q, p$, when $u_{q}-u_{p}$ becomes $K-u_{q}+u_{p}$.

The relation (4.30) manifests the difference property. It has far-ranging consequences, in fact it enables us to calculate the spontaneous magnetization, i.e. the order parameter (3.17) with $j=1$. [4, chapter 13]

To see this, note that we write the first equation (3.25) as

$$
A_{1}^{a}\left(u_{q}-u_{p}\right)=\left[X\left(u_{p}\right)^{a}\right]^{-1} X\left(u_{q}\right)^{a} .
$$

Replacing $p, q$ by $q, r$ and multiplying, this gives

$$
A_{1}^{a}\left(u_{q}-u_{p}\right) A_{1}^{a}\left(u_{r}-u_{q}\right)=A_{1}^{a}\left(u_{r}-u_{p}\right)
$$

i.e.

$$
A_{1}^{a}(u) A_{1}^{a}(v)=A_{1}^{a}(u+v),
$$


for arbitrary $u, v$. Interchanging $u, v$, it follows that

$$
A_{1}^{a}(u) A_{1}^{a}(v)=A_{1}^{a}(v) A_{1}^{a}(u),
$$

so $A_{1}^{a}(u), A_{1}^{a}(v)$ commute, for all $u, v$.

There is therefore a similarity transformation, independent of $u$, that diagonalises $A_{1}^{a}(u)$. Let us assume this transformation has been applied and take $A_{1}^{a}(u)$ to be diagonal. Then it follows from (4.35) that each diagonal element of $A_{1}^{a}(u)$ is an exponential function of $u$, i.e.

$$
\left[A_{1}^{a}(u)\right]_{i, i}=\exp \left(-n_{i} u\right),
$$

where $n_{i}$ is some number.

Allow $u=u_{q}-u_{p}$ to be an arbitrary complex number. Since

$$
\operatorname{scd}\left(u+21 K^{\prime}\right)=-\operatorname{scd}(u),
$$

it follows from (4.28) that incrementing $u$ by $2{ }_{1} K^{\prime}$ merely negates $W_{p q}(1)$ and $\bar{W}_{p q}(1)$. From series expansions, we expect $A_{1}^{a}(u)$, and indeed all the functions we have defined in the thermodynamic limit of a large lattice, to be an analytic function of $u$ within the extended physical domain $0<\Re(u)<K$, which implies

$$
A_{1}^{a}\left(u+2 K^{\prime}\right)=(-1)^{a} A_{1}^{a}(u) .
$$

It follows that

$$
n_{i}=\pi m_{i} /\left(2 K^{\prime}\right)
$$

where $m_{i}$ is an even integer for $a=0$, an odd integer for $a=1$.

If we take the low-temperature limit $k^{\prime} \rightarrow 0$, keeping $u$ in the vicinity of $K / 2$, then to leading order in $q^{\prime}=\mathrm{e}^{-\pi K / K^{\prime}}$,

$$
W_{p q}(1)=\mathrm{e}^{-\pi u /\left(2 K^{\prime}\right)}, \bar{W}_{p q}(1)=\mathrm{e}^{\pi(u-K) /\left(2 K^{\prime}\right)} .
$$

We find that $A_{1}^{a}(u)$, as originally defined before (2.4), is already diagonal to leading order. Let the spins to the left of and including $a$ in Fig. 2 be $\ldots, \lambda_{2}, \lambda_{1}, a$, where $\lambda_{1}$ is next to $a, \lambda_{2}$ is next to $\lambda_{1}$, etc. Then the corresponding diagonal element of $A_{1}^{a}(u)$ is indeed of the form (4.37), (4.39), the index $i$ being replaced by the spin set $\lambda$,i.e.

$$
A_{1}^{a}(u)_{\lambda, \lambda}=\exp \left\{-\pi u m_{\lambda} /\left(2 K^{\prime}\right)\right\},
$$


where

$$
m_{\lambda}=\left|a-\lambda_{1}\right|+3\left|\lambda_{1}-\lambda_{2}\right|+5\left|\lambda_{2}-\lambda_{3}\right|+\cdots \quad .
$$

We have obtained this result in the low-temperature limit $k^{\prime} \rightarrow 1$. However, for all $k^{\prime}$ the $m_{\lambda}$ must be integers and we do not expect them to change discontinuously as $k^{\prime}$ increases to unity. Hence we expect the formulae (4.41), (4.42) for the diagonal elements of $A_{1}^{a}(u)$ to be exact for $0<k^{\prime}<1$.

Set $u_{1}=u_{2}=u_{q}-u_{p}$ and $u_{2}=u_{4}=K-u_{q}+u_{p}$. Then from (3.22),

$$
A_{i}^{a}(p, q)=A_{1}^{a}\left(u_{i}\right)
$$

so these matrices, together with the product

$$
M^{a}=A_{1}^{a}(p, q) A_{2}^{a}(p, q) A_{3}^{a}(p, q) A_{4}^{a}(p, q)
$$

are diagonalised by the same transformation that diagonalizes $A_{1}^{a}(u)$, and

$$
M_{\lambda, \lambda}^{a}=x^{m_{\lambda}}
$$

where $x=q^{\prime}=\mathrm{e}^{-\pi K / K^{\prime}}$.

These are also the elements of the diagonalized matrix $\mathcal{M}^{a}$ : we see that our calculation is consistent with the independence of $\mathcal{M}^{a}$ on the rapidity variables $p, q$.

It is now straightforward to calculate the spontaneous magnetization from (3.17). First set $\lambda_{0}=a$ and

$$
l_{i}=\left|\lambda_{i}-\lambda_{i+1}\right|, \quad i \geq 0,
$$

so $l_{i}=0$ if $\lambda_{i}=\lambda_{i+1}$, otherwise $l_{i}=1$. Take $\lambda_{i}=0$ for sufficiently large $i$, then to modulo 2 ,

$$
a=\lambda_{0}+\lambda_{1}+\lambda_{2}+\cdots .
$$

and

$$
m_{\lambda}=\lambda_{0}+3 \lambda_{1}+5 \lambda_{2}+\cdots .
$$

From (3.17) and (4.45),

$$
\left\langle(-1)^{a}\right\rangle=\sum_{l}(-1)^{a} x^{m_{\lambda}} / \sum_{l} x^{m_{\lambda}} .
$$


Here $a, m_{\lambda}$ are the linear expressions immediately above and the sums are over $l_{0}, l_{1}, l_{2}, \ldots=0,1$, without restriction. Each sum therefore factors into a product of individual sums over $l_{0}, l_{1}, l_{2}, \ldots$, giving

$$
\left\langle(-1)^{a}\right\rangle=\frac{(1-x)\left(1-x^{3}\right)\left(1-x^{5}\right) \cdots}{(1+x)\left(1+x^{3}\right)\left(1+x^{5}\right) \cdots} .
$$

Using known elliptic function formulae,[16, 8.197.4] and [4, 15.1.4b] the RHS is $k^{1 / 4}$, so

$$
\left\langle(-1)^{a}\right\rangle=k^{1 / 4}=\left(1-k^{\prime 2}\right)^{1 / 8}
$$

which is the famous result announced by Onsager in 1949[23] and derived by Yang in 1952.[26]

\section{Summary}

We have expicitly shown how the star-triangle relation implies that the corner transfer matrices factor, causing the reduced matrix functions $\mathcal{A}_{i}^{a}, \mathcal{B}_{i}, \mathcal{F}_{i}^{a}, \mathcal{G}_{i}^{a}$ to depend on at most one of the two rapidity variables. The rotation (and reflection) relations enable one to express the matrices, for $i=1, \ldots, 4$, in terms of the single $i=1$ case.

Curiously, this by itself does not seem enough to enable one to calculate quantities such as the order parameters: if one also has the rapidity difference property (4.27), then the corner transfer matrices commute and can be simultaneously reduced to diagonal form. The eigenvalues are necessarily exponentials in $u=u_{q}-u_{p}$ and are periodic of period $2{ }_{1} K^{\prime}$ : this means they are defined by certain integers $m_{\lambda}$, and these integers can be obtained from a low-temperature limit. The order parameters follow immediately.

It still seems strange that the method should fail for a model such as the chiral Potts model, which does satisfy the star-triangle relation, so in that sense is "solvable". As yet the method has only yielded finite series expansions. [6, [7]

The order parameters have now been obtained by a specialization of the Jimbo, Miwa, Nakayashiki method,[18 - [1] but it would be interesting to rescue the corner transfer matrix technique for this model. For the models with the difference property, diagonalizing the matrices leads directly to the Jacobi elliptic parametrization. For the chiral Potts model, is there any way of expressing the matrices that leads to an analogous parametrization? 


\section{References}

[1] Baxter R J 1968 Dimers on a rectangular lattice J. Math. Phys. 9650 $-654$

[2] Baxter R J 1977 Corner transfer matrices of the eight-vertex model. II. The Ising case, J. Stat. Phys. $171-14$

[3] Baxter R J 1981Corner transfer matrices Physica 106A 18 - 27

[4] Baxter R J 1982 Exactly Solved Models in Statistical Mechanics Academic, London

[5] Baxter R J 1980 Hard hexagons: exact solution J. Phys. A 13 L61 - L70

[6] Baxter R J 1991 Corner transfer matrices of the chiral Potts model $J$. Stat. Phys. $63433-453$

[7] Baxter R J 1993 Corner transfer matrices of the chiral Potts model. II. The triangular lattice J. Stat. Phys. $70535-582$

[8] Baxter R J 1998 Functional relations for the order parameters of the chiral Potts model J. Stat. Phys. 91499 - 524

[9] Baxter R J 1999 Planar lattice gases with nearest-neighbour exclusion Annals Comb. $3191-203$

[10] Baxter R J 2005 Derivation of the order parameter of the chiral Potts model Phys. Rev. Lett. 94130602 (3pp)

[11] Baxter R J 2005 The order parameter of the chiral Potts model J. Stat. Phys. $1121-36$

[12] Baxter R J and Enting I G 1979 Series expansions from corner transfer matrices: the square lattice Ising model J. Stat. Phys. 21 103-123

[13] Baxter R J, Perk J H H and Au-Yang H 1988 New solutions of the star-triangle relations for the chiral Potts model Phys. Lett. A 128138 142

[14] Baxter R J and Tsang S K 1980 Entropy of hard hexagons J. Phys. A $131023-1030$ 
[15] Gaudin M 1991 La relation étoile-triangle d'un modèle elliptique $Z_{N} \mathrm{~J}$. de Physique I 1 351-361

[16] Gradshteyn I S and Ryzhik I M 1965 Table of integrals, series and products Academic Press (NY)

[17] Hasegawa K and Yamada Y 1990 Algebraic derivation of the broken $Z_{N}$ symmetric model Phys. Lett. A146 387 - 396

[18] Jimbo M, Miwa T and Nakayashiki A 1993 Difference equations for the correlation functions of the eight-vertex model J. Phys. A $262199-2210$

[19] Kashiwara M and Miwa T 1986 A class of elliptic solutions to the startriangle relation Nucl. Phys. B275 [FS17] 121-134

[20] Kasteleyn P W 1963 Dimer statistics and phase transitions J. Math. Phys. $4287-293$

[21] Kaufman B 1949 Crystal statistics. II. Partition function evaluated by spinor analysis Phys. Rev. $761232-1243$

[22] Montroll E W, Potts R B and Ward J C 1963 Correlations and spontaneous magnetization of the two-dimensional Ising model J. Math. Phys. $4308-322$

[23] Onsager L 1949 Nuovo Cimento 6 (suppl.) 261

[24] Sykes M F, Gaunt D S, Martin J L and Mattingly S R 1973 Derivation of low-temperature expansions for Ising model. IV. Two-dimensional lattices - temperature grouping J. Math Phys. 141071 - 1074

[25] Tsang S K 1977 Square lattice variational approximations applied to the Ising model J. Stat. Phys. $2095-114$

[26] Yang C N 1952 The spontaneous magnetization of a two-dimensional Ising model Phys. Rev. $85808-816$ 